\title{
Reconstructing the Three-Dimensional Point Cloud of a Draped Fabric Based On a Two-Dimensional Projection
}

\author{
Zhicai Yu, Yueqi Zhong, and Haoyang Xie
}

\begin{abstract}
In order to reconstruct the three-dimensional (3D) point cloud of a draped fabric based on a two-dimensional fabric drape projection, the three-dimensional point clouds of the draped fabrics were scanned with a self-built 3D scanning device. A resampling method based on local linear embedding (LLE) was used to represent different $3 D$ point clouds with the same point number and point sequence. Principal Component Analysis (PCA) was used to reduce the dimension of the resampled 3D point clouds. With PCA, a completed resampled point cloud could be represented with a signature of length fifty-seven. At last, a regression model with a two- dimensional (2D) fabric drape projection as input was constructed and trained to predict the signature of length fifty-seven. With the predicted signature, the 3D point cloud of a draped fabric could be reconstructed. The result shows that all resampled $3 \mathrm{D}$ point clouds of draped fabric have the same point number and point sequence. The errors between the reconstructed 3D point clouds and the ground truth are all within $6.92 \mathrm{~mm}$.
\end{abstract}

Index Terms-Draped fabric, 3D triangular mesh, 2D projection, PCA, deep learning.

\section{INTRODUCTION}

Fabric drape is an aspect of the fabric style. As for the evaluation of fabric drape performance, the research object can be either a 2D projection [1] or a 3D model of a draped fabric. The $2 \mathrm{D}$ projection is easy to operate, and the $3 \mathrm{D}$ model contains more information than the $2 \mathrm{D}$ projection does With the development of 3D scanning devices and technology, the cost of obtaining a 3D model of a draped fabric is getting lower and lower, which promotes the study on fabric drape performance with 3D models. For example, Wang et al [2] proposed a method to identify the contour information in the panoramic raster image. With the method, the grating stripes of a draped fabric were located. Finally, the height of the grating stripes was inferred. Mah et al [3] scanned the $3 \mathrm{D}$ configuration of a draped fabric via a $3 \mathrm{D}$ human body scanner. The distribution of the distance between the draped fabric and the supporting cylinder was studied. Shi et al [4] designed a new system for reconstructing the $3 \mathrm{D}$ configuration. The system can test

Manuscript received July 13, 2020; revised February 1, 2021. This work was supported by the National Natural Science Foundation of China (Grant No. 61572124) and the Special Excellent Ph.D. International Visit Program by Donghua University (DHU).

Zhicai Yu and Haoyang Xie are with the College of Textiles, Donghua University, Shanghai 201620, China (e-mail: zhicai-yu@qq.com; xie_haoyang@qq.com).

Yueqi Zhong is with College of Textiles, Donghua University, Shanghai 201620, China and the Key Laboratory of Textile Science \& Technology of Ministry of Education, College of Textiles, Donghua University, Shanghai, 201620, China (corresponding author; e-mail: zhyq@dhu.edu.cn). various samples without switching the background of a draped fabric.

Hu et al. [5] proposed a simple and effective method for fabric drape reconstruction. Their method can reconstruct the 3D color model of draped fabric based on the image sequence acquired by the rear lens of a mobile phone. The reports mentioned above strongly demonstrated the importance of 3D fabric drape models. However, there is a commonality for these reports, i.e., they cannot consider both the device simplicity and the short processing flow simultaneously. In Hu's research, a 3D point cloud is generated from a sequence of images acquired by a mobile phone. However, their method requires a long time for the $3 \mathrm{D}$ reconstruction. In addition, manual scaling and de-noising are necessary for their method. Besides, the point number and triangular topology of scanned models are different, which is unfavorable for the comparison of different drape models.

Therefore, this study is aimed to reconstruct the $3 \mathrm{D}$ point cloud of a draped fabric based on a $2 \mathrm{D}$ projection. Meanwhile, different reconstructed 3D point clouds have the same point number and point sequence. The organization of the rest paper was explained as follows.

\section{MATERIAL AND METHODS}

\section{A. Preparing Fabric Samples}

Fifty-one fabrics with various fabric hand and fabric style collected from the Chinese market were washed and ironed to remove wrinkles on the fabric surface. Circular specimens of radius $120 \mathrm{~mm}$ were cut from each fabric. The center of each circular specimen was marked. The circular fabric specimens were conditioned for 48 hours at a constant temperature and humidity laboratory (temperature: $23 \pm 2^{\circ} \mathrm{C}$, humidity: $65 \% \pm 2 \%)$.

\section{B. Scanning Device}

The 3D scanning device for fabric drape is constructed with four depth cameras by referring to the method of $\mathrm{Wu}$ et al [6]. The structure of the scanning device is shown in Fig. 1a. The fabrics were centered on a supporting disk with a radius of $60 \mathrm{~mm}$ surrounding by four RGB-Depth cameras. The angle between two adjacent cameras is $90^{\circ}$. To reach an accurate scanning result, a T-shaped checkerboard as shown in Fig. 1b was used to calibrate the extrinsic parameters of the four cameras based on the algorithm proposed by Zhang et al [7] and Wu et al [7]. Fig. 1c demonstrates four point-cloud patches of draped fabric captured by each camera. It is observed that all four point-cloud patches of draped fabric were incomplete. They were fused to generate a complete point cloud, as shown in Fig. 1d. The corresponding triangulated mesh was generated with surface reconstruction, 
as shown in Fig. 1e. All fifty-one fabrics were draped one hundred times separately. Therefore 5100 three-dimensional triangular meshes of draped fabrics were obtained in this study.

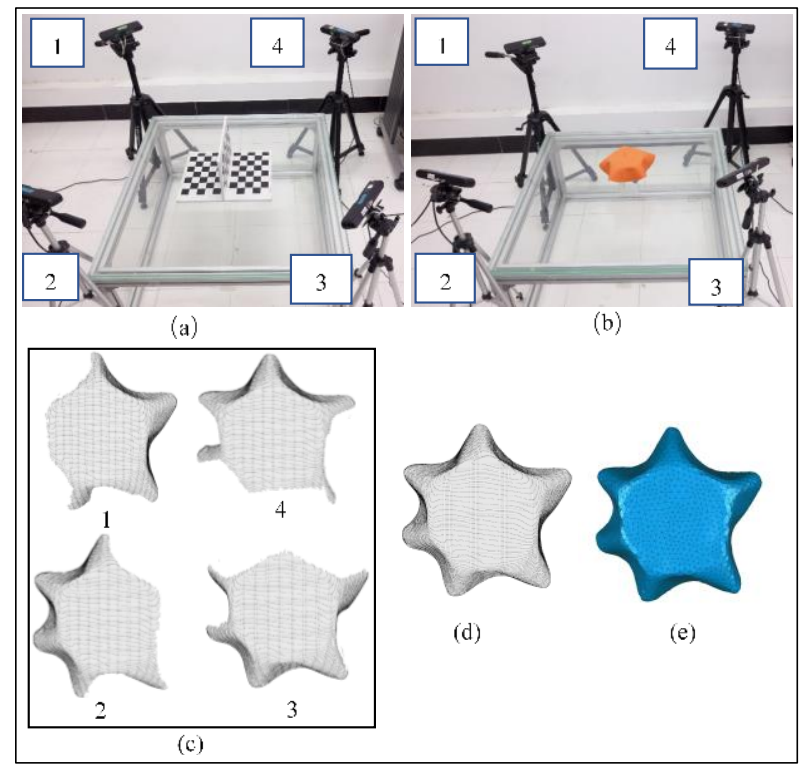

Fig. 1. The pipe of scanning fabric drape (a) scanning device. (b) Calibration. (c) four patches (d) completed 3D points cloud. (e) 3D triangular mesh.

\section{Resampling the 3D Draped Fabrics}

The 3D triangular meshes of different fabrics samples or different fabric drape experiments obtained in Section B have different point numbers and triangle topology. It is difficult to compare 3D triangular meshes with different point number and point sequence, i.e., the original 3D triangular meshes are lack of comparability. Therefore, it is necessary to resample different triangular meshes to make them the same point number and point sequence. A new method based on locally linear embedding (LLE [8]) is proposed to resample the 3D triangular mesh of a draped fabric. The detail of the method was shown as following.

Step 1. The total length (L3D) of the closed 3D boundary of the 3D triangular mesh (Fig. 2a) of a draped fabric was measured. The discrete points on the 3D boundary of draped fabric were mapped into a two-dimensional circle (Fig. 2b) sequentially. The perimeter of the circle is equal to the total length of the $3 \mathrm{D}$ boundary. Besides, the point sequence in the two-dimensional circle is equal to the point sequence in the

\section{D boundary.}

Step 2. LLE algorithm was used to reduce the dimension of the 3D triangular mesh. The mapped points on the circle (Fig. 2b) were used as a constraint. The result of LLE was a triangular mesh with the shape of circle as shown in Fig. 2c. It has the same point number and triangle sequence with the original 3D triangular mesh (Fig. 2a).

Step 3. Regular points within a circle of radius $120 \mathrm{~mm}$ (as shown in Fig. 2d) were generated. The result of LLE algorithm (Fig. 2c) and the generated regular points (Fig. 2d) were overlapped, as shown Fig. 2e. It is observed that each point in the generated regular points was within a certain triangle of the $2 \mathrm{D}$ triangular mesh.

The correspondence of a triangle in the raw 3D triangular mesh (Fig. 2a) and its mapping in the flatten 2D triangular mesh (Fig. 2c) were shown in Fig. 3.

As shown in Fig. 3, T1 refers to a triangle in the raw 3D triangular mesh (Fig. 2a). T2 refers to a triangle in the flatten 2D triangular mesh (Fig. 2c). T1 has the same triangle index as $\mathrm{T} 2$. If point $\mathrm{Q}$ is one of the points in $\mathrm{T} 2$. The mapping of point $\mathrm{Q}$ in $\mathrm{T} 1$ is $\mathrm{P}$. The point $\mathrm{P}$ could be presented with the point $\mathrm{P} 1, \mathrm{P} 2$ and $\mathrm{P} 3$, i.e.,

$$
P=m \times P_{1}+n \times P_{2}+(1-m-n) \times P_{3}
$$

With the given point $\mathrm{P}$, the weights $\mathrm{m}$ and $\mathrm{n}$ in Equation (1) could be inferred according to Equation (1). Sze \& Liu [9] demonstrated that fabric can deform under its gravity. The deformation result from bending and shearing mostly. The deformation resulted from the stretch during fabric draping is quite small. It can be ignored. Therefore, the result of LLE is very close to the circular fabric sample before draping. The most important is that the result of LLE has the same point number and topology with the original 3D triangular mesh. Therefore, the mapped point $\mathrm{Q}$, which is the map of point $\mathrm{P}$, could be inferred with Equation (2).

$$
Q=m \times Q_{1}+n \times Q_{2}+(1-m-n) \times Q_{3}
$$

Because different 3D triangular meshes were resampled with the same regular 2D points (Fig. 2d), all resampled point clouds have the same point number, i.e., each resampled $3 \mathrm{D}$ point cloud in this study has $48193 \mathrm{D}$ points because there are 4819 points in Fig. 2d. Besides, all resampled point clouds have the same point sequence.

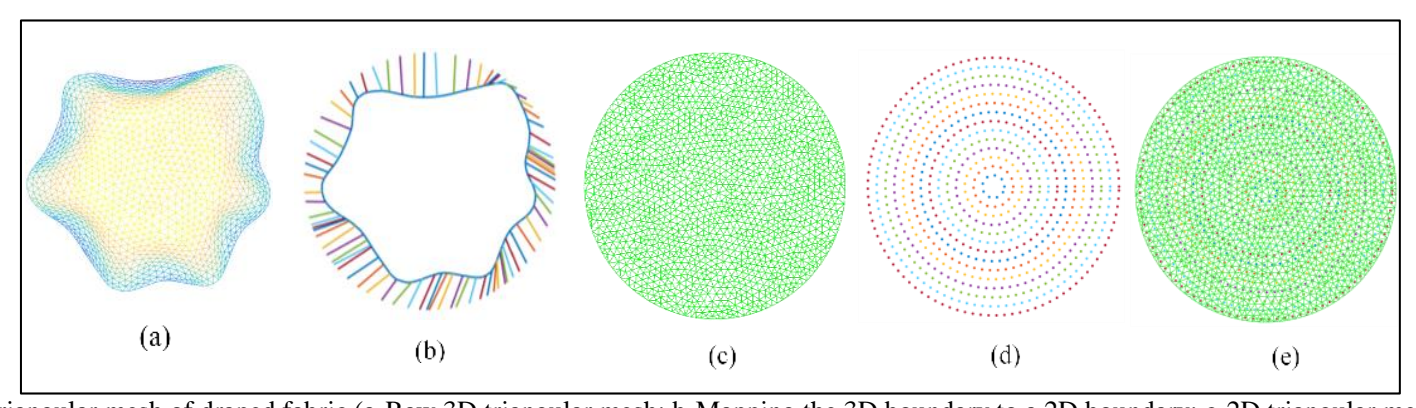

Fig. 2. The triangular mesh of draped fabric (a-Raw 3D triangular mesh; b-Mapping the 3D boundary to a 2D boundary; c-2D triangular mesh; d-Regular points, e-Resampling).

\section{Reducing the Dimension of the Resampled $3 D$ Point Cloud with PCA}

As shown above, there are 4819 vertices for each resampled 3D point cloud. With flattening, each 3D point cloud was converted to a vector of length $14457(4819 \times 3)$. It is difficult to train a regression model which has an output of length 14457 . Besides, there is a high correlation between 
different points in the same 3D point cloud. In order to reduce the high correlation between different points in the same 3D point cloud, the dimension of the whole $3 \mathrm{D}$ point cloud should be reduced, i.e., to map the completed 3D point cloud into a signature with a low dimension. Principle Component Analysis (PCA) is an efficient and reversible dimension reduction method. Therefore, PCA was used in this study to map the $3 \mathrm{D}$ point cloud into a new space.

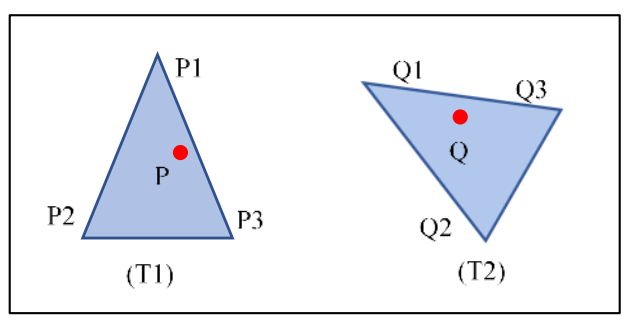

Fig. 3. The schematic diagram of the 3D triangle and the corresponding mapped triangle.

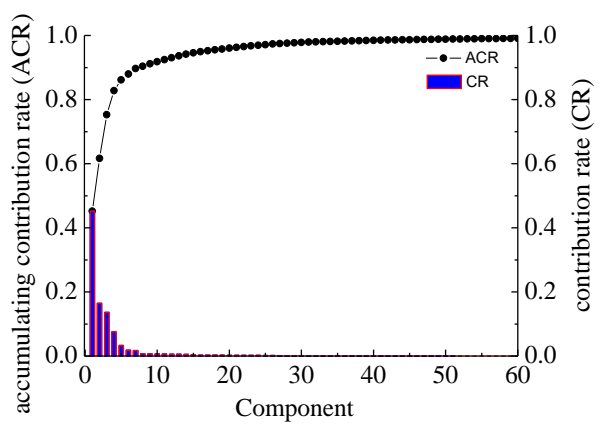

Fig. 4. The accumulating contribution rate (ACR) and contribution rate (CR).

Principal component analysis (PCA) is a statistical procedure that uses an orthogonal transformation to convert a set of observations of possibly correlated variables into a set of values of linearly uncorrelated variables called principal components. The number of distinct principal components is equal to the smaller of the number of original variables or the number of observations minus one. To determine the signature dimension, i.e., the number of principal components, we computed the component variance of different principal components for the whole 3D point clouds The accumulating contribution rate (ACR) and contribution rate (CR) was shown in Fig. 4.

As shown in Fig. 4, with the increase of the serial number of the principal component, its corresponding component variance decreases. To maintain the majority of features, the first fifty-seven principal components were selected. The accumulating contribution rate (ACR) of the selected fifty-seven principal components reached $99.02 \%$. That is to say, the length of the signature for PCA reconstruction is determined as fifty-seven.

\section{E. Predicting the Signature for PCA Reconstruction}

The key to PCA reconstruction is predicting the signature of length fifty-seven. Once we obtain the signature, the completed 3D point cloud of a draped fabric could be reconstructed. In this study, the projection image of a draped fabric was used as the input. The signature for PCA reconstruction is used as the output. A raw 3D triangular mesh obtained in Section II.B was shown as Fig. 5a.

To improve the robustness of the proposed method, nine derivations of Fig. 5a were generated by scaling and translating Fig. 5a randomly ten times. The binary images of the ten derivations were rendered. Three of the ten binary images were shown as Fig. 5b, Fig. 5c and Fig. 5d. Therefore, there are ten binary images, which correspond to a 3D point cloud, i.e., a real signature. When fabricating the training specimen, one of the ten binary images was selected from the ten binary images as the input of the 3D triangular mesh (Fig. 5a).

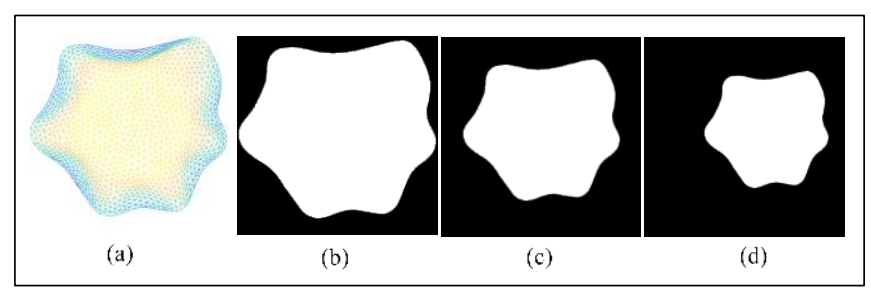

Fig. 5. The 3D triangular mesh and the 2D projection of draped fabric.

In this study, a convolutional neural network, VGG16[10], was constructed to bridge the $2 \mathrm{D}$ projection and the signature for PCA reconstruction. By repeatedly stacking $3 \times 3$ small convolution kernels and $2 \times 2$ maximum pooling layers, VGG16 successfully constructed a deep convolutional neural network with 16 layers. Besides, VGG16 has strong portability, and it can be used to classification, segmentation, and regression. The structure of VGG16 is shown in Fig. 6.

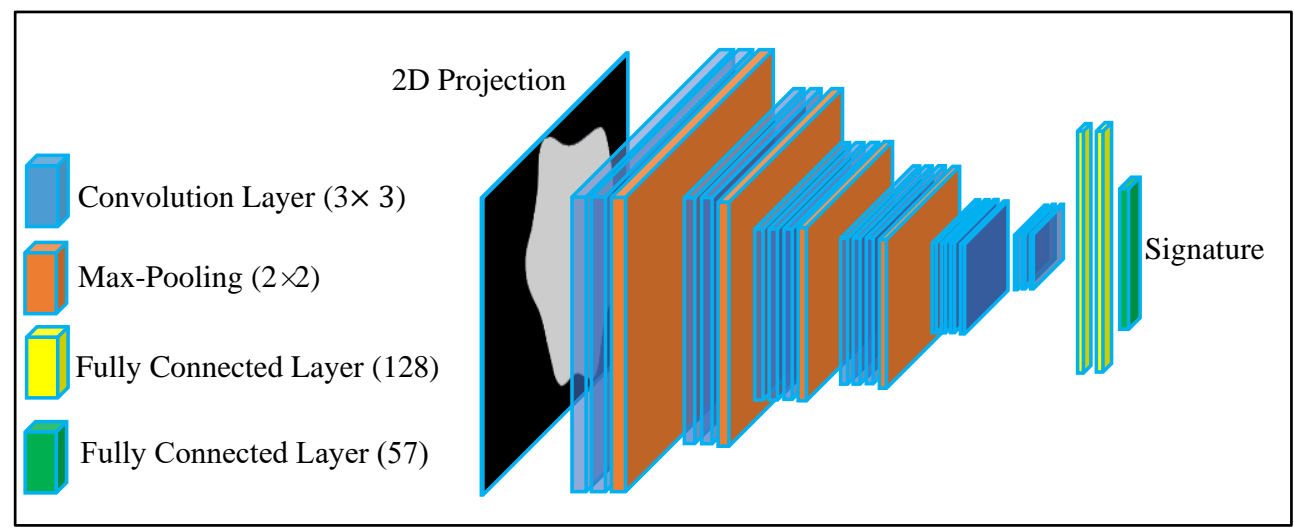

Fig. 6. The structure of VGG16 for regression.

It is observed that the $2 \mathrm{D}$ projection of a draped fabric was used as the input of VGG16. The binary image size is
$128 \times 128$. Several blocks combined with the convolution layer and max-pooling were repeated to extract the feature 
map. The kernel size of the convolutional layers is $3 \times 3$. The kernel size of the max-pooling layers is $2 \times 2$. Followed with two fully-connected layers (128 nodes), the signature of length fifty-seven was predicted. The activation function in the fully-connected layers was 'ReLU'. The loss function used in the regression model is the mean-squared error.

4500 samples were selected randomly from the 5100 resampled 3D point clouds of 51 different fabrics as training samples. The rest 600 samples were used as the testing samples. We implement all training and testing of this study on a laptop with four cores processor at $2.8 \mathrm{GHz}$ and $16 \mathrm{~GB}$ RAM.

\section{RESUlTS AND DisCUSSION}

\section{A. The Errors of the Triangle Area Resulted from the LLE Method}

As shown in Fig. 7, the raw triangular mesh of draped fabric was shown as Fig. 7a. With flattening, the area of triangles in the raw triangular mesh may change. The comparative error of the triangle area after flattening was used to evaluate the LLE method. The flatten 2D triangular mesh with a hot-map of comparative error was shown as Fig. $7 b$.

It is observed that most of the errors are less than $15 \%$. There are only several triangles, which have comparative errors of more than $15 \%$. These triangles are mostly located near the boundary. The results demonstrate that it is reasonable to flatten the $3 \mathrm{D}$ triangular mesh with the LLE method.

\section{B. The Resampled 3D Point Clouds and the Reconstructed 3D Point Cloud Based on Real Signatures}

The resampled 3D point clouds and the reconstructed 3D point cloud were shown as Fig. 8.

As shown Fig. 8, the blue ones are the raw resampled point clouds and the orange ones are the reconstructed point clouds. The results show that when the dimension of the signatures for PCA reconstruction is fifty-seven, the reconstructed 3D point clouds and the resampled point clouds were very close. As for the objective evaluation, the maximum distances between the resampled point clouds and the reconstructed point clouds were within $3.51 \mathrm{~mm}$. The result proved the feasibility of reconstructing the 3D point cloud of a draped fabric with PCA reconstruction.

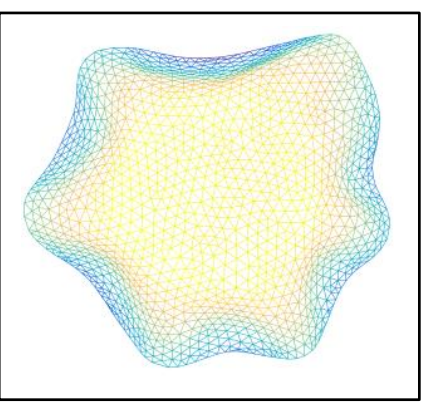

(a)

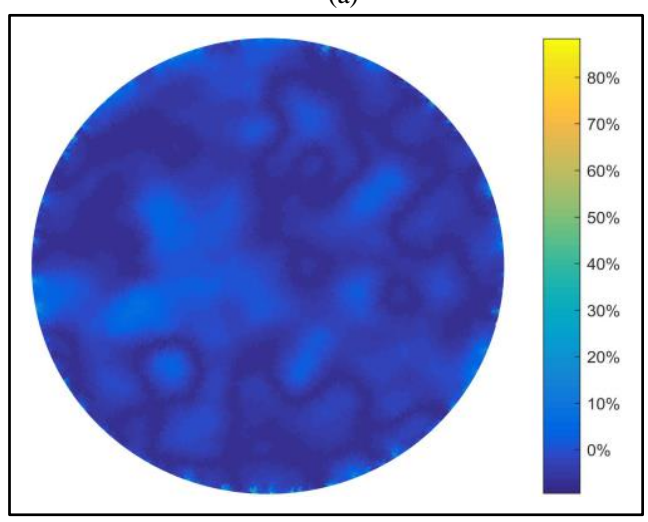

(b)

Fig. 7. The comparative errors of triangle area resulted from the LLE method (a-The raw 3D triangular mesh; b-The flatten 2D triangular mesh with hot-map).
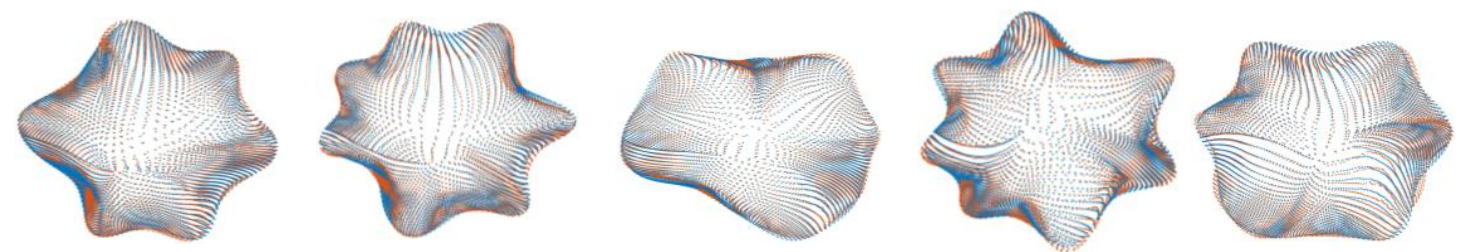

Fig. 8. The resampled 3D point clouds and the reconstructed 3D point clouds based on real signature.

\section{Results of Training the Regression Models}

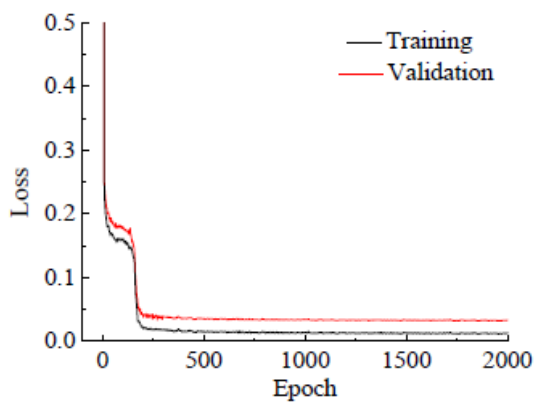

Fig. 9. The relationship between training epoch and loss.

The relationship between training epochs and loss is shown in Fig. 9. It is obvious that the loss converges fast. The validation loss was close to the training loss. This demonstrated that the regression model is suitable for predicting the signature for PCA reconstruction.

\section{The 3D Point Clouds Reconstructed Based on the Predicted Signatures}

The 3D point clouds reconstructed based on the predicted signatures and the raw resampled point clouds were shown as Fig. 10. The blue samples are the raw resampled point clouds. The orange samples are the point clouds reconstructed based on the predicted signatures. It is observed that the reconstructed 3D point clouds were close to the raw resampled point clouds. The objective evaluations show that the maximum errors of the 3D points in the 600 testing samples were all within $6.92 \mathrm{~mm}$. The obvious errors of the reconstructed 3D point clouds in Fig. 10 may result from two aspects. Firstly, although the signature of length fifty-seven could represent a completed $3 \mathrm{D}$ point cloud of a draped fabric, 
a lot of details were still ignored because of PCA. Secondly, the signature for PCA reconstruction is a vector of length fifty-seven. Although the convolutional neural network could extract the high-level feature of the images, it is hard to predict the signatures completely. That is to say, it is easy to introduce new errors for the predicted signatures during the training and predicting. Inspired by the results, further work may be conducted to reduce the reconstructing pipeline.

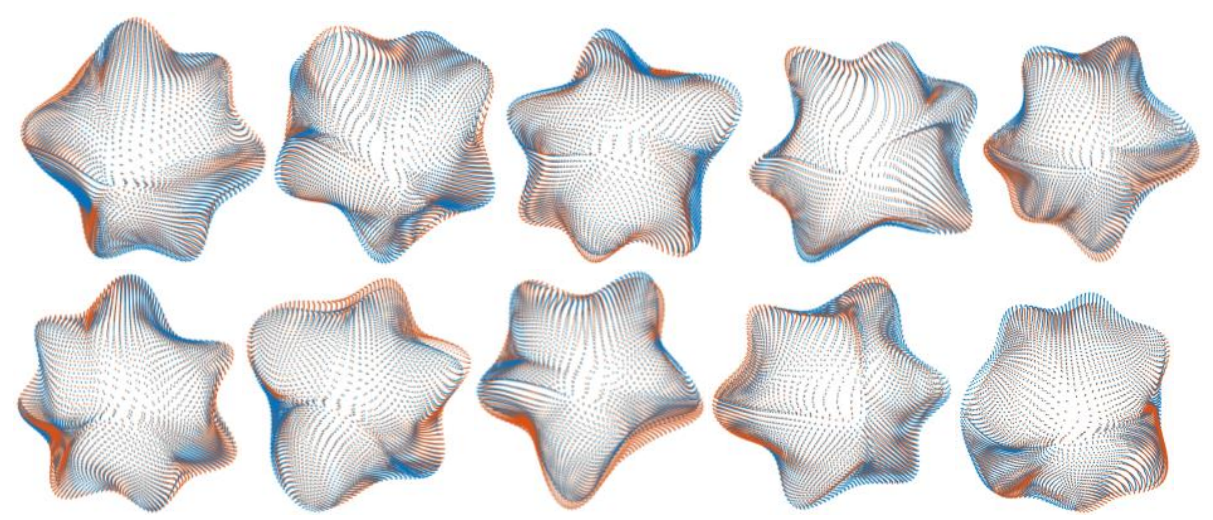

Fig. 10. The 3D point clouds reconstructed based on the predicted signatures.

\section{CONCLUSION}

In this study, a new method was proposed to reconstruct the $3 \mathrm{D}$ point cloud of draped fabric based on a $2 \mathrm{D}$ projection. Two conclusions were drawn: (1) The resampled 3D point clouds could represent the raw triangular meshes accurately. Besides, different resampled 3D point clouds have the same point number and point topology, which is beneficial to the post-processing. (2) The proposed method could bridge the $2 \mathrm{D}$ projection and the signature for PCA reconstruction. The reconstructed $3 \mathrm{D}$ point cloud based on the predicted signature is close to the ground-truth. The method proposed in this paper could provide an efficient approach to reconstruct the $3 \mathrm{D}$ point cloud based on the $2 \mathrm{D}$ images obtained with the traditional 2D fabric drape testers. Further work may be conducted to apply more advanced regression models and reduce the reconstructing pipeline.

\section{CONFLICT OF INTEREST}

The authors declare no conflict of interest.

\section{AUTHOR CONTRIBUTIONS}

Zhicai Yu prepared the fabric samples, constructed the 3D fabric scanning device, finished the reconstructing of $3 \mathrm{D}$ fabric drape models, analyzed the data and wrote the paper. Yueqi Zhong supervised the experimental and revised the paper. Haoyang Xie conducted the fabric scanning and data analysis. All authors had approved the final version.

\section{REFERENCES}

[1] X. F. Yu, J. Liu, and Y. Z. Fei, “Applying image processing technique to study the fabric drape performance-The method of measuring the fabric drape," Journal of China Textile University, vol. 25, no. 1, pp. 33-37, February 1999.

[2] S. B. Wang, H. Zou, Y. Shen, and X. M. Liu, "Three-dimensional shape reconstruction algorithm of fabric drapability based on raster imaging," Journal of Engineering Graphics, vol. 29, pp. 136-141, February 2008.

[3] T. Mah and G. W. Song, "An investigation of the assessment of fabric drape using three-dimensional body scanning," Journal of the Textile Institute, vol. 101, pp. 324-335, March 2010.

[4] Z. P. Shi, "Software system research and development of the fabric drape ability 3-Dimensional tester," Master dissertation, Dalian Maritime University, Da Lian, China, 2007.
[5] K. Hu, "Reconstruction and measurement of fabric drape based on an image sequence," Master dissertation, Donghua University, Shanghai, China, 2016

[6] G. Wu, "Three-dimensional reconstruction and shape analysis of human feet based on multiple depth cameras," Ph.D. dissertation, Donghua University, Shanghai, China, 2017.

[7] Z. Y. Zhang, "A flexible new technique for camera calibration," IEEE Computer Society, vol. 22, pp. 1330-1334, December 2000.

[8] X. L. Teng, B. Wu, and W. W. Yu, "A hand gesture recognition system based on locally linear embedding," Journal of Visual Languages and Computing, vol. 16, no. 5, pp. 442-454, October 2005.

[9] K. Y. Sze and X. H. Liu, "A co-rotational grid-based model for fabric drapes," International Journal for Numerical Methods in Engineering. vol. 57, pp. 1503-1521, May 2003.

[10] S. Karen, "Very deep convolutional network for large-scale image recognition," arXiv:1409.1556v6 [cs.CV], 2015.

Copyright (C) 2021 by the authors. This is an open access article distributed under the Creative Commons Attribution License which permits unrestricted use, distribution, and reproduction in any medium, provided the original work is properly cited (CC BY 4.0).

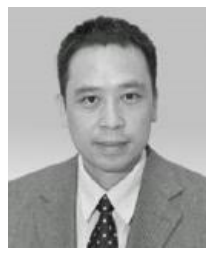

Zhicai Yu received a master's degree in textile material and textile design from Zhongyuan University of Technology, Zhengzhou, China, in 2015. He is currently pursuing a Ph.D. degree at Donghua University, Shanghai, China. His major is digital textile engineering. His work related to fabric drape, 3D scanning technology, image processing, machine learning, deep learning, fabric inquiry and analysis.

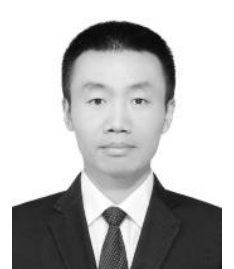

Yueqi Zhong is now a professor in the College of Textiles, Donghua University. He received a master's degree in textile engineering from Xi'an Polytechnic University, in 1998, and the Ph.D. degree in textile production design from Donghua University, in 2001. He was a postdoctoral fellow at the University of Texas at Austin (2001-2005). His research interests include virtual garments, physically-based cloth simulation, geometry processing, interactive techniques, and deep learning.

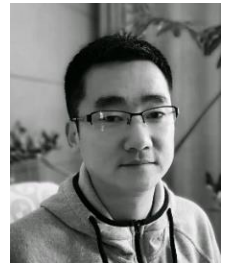

Haoyang Xie received a master's degree in information assurance from the Fort Hays State University, KS, US, in 2011. He is currently pursuing a Ph.D. degree at Donghua University, Shanghai, China. His current research interests include digital geometry processing, image processing, machine learning, and deep learning. 